Math. Model. Nat. Phenom.

Vol. 5, No. 2, 2010, pp. 100-124

DOI: $10.1051 / \mathrm{mmnp} / 20105204$

\title{
Noise Shaping in Neural Populations with Global Delayed Feedback
}

\author{
O. Ávila Åkerberg ${ }^{1}$ and M. J. Chacron ${ }^{1,2, *}$ \\ ${ }^{1}$ Department of Physics, McGill University, Montreal, H3G 1Y6, Canada \\ 2 Department of Physiology, McGill University, Montreal, H3G 1Y6, Canada
}

\begin{abstract}
The interplay between intrinsic and network dynamics has been the focus of many investigations. Here we use a combination of theoretical and numerical approaches to study the effects of delayed global feedback on the information transmission properties of neural networks. Specifically, we compare networks of neurons that display intrinsic interspike interval correlations (nonrenewal) to networks that do not (renewal). We find that excitatory and inhibitory delays can tune information transmission by single neurons but not by the entire network. Most surprisingly, addition of a delay can change the dependence of the information on the coupling strength for renewal neurons and not for nonrenewal neurons. Our results show that intrinsic ISI correlations can have nontrivial interactions with network-induced phenomena.
\end{abstract}

Key words: information theory, neural networks, nonrenewal, delay

AMS subject classification: 68P30, 92B20, 34K50

\section{Introduction}

There is considerable interest in understanding the properties of excitable systems $[5,25,54,73$, 75] and in particular neural spike trains. The study of experimental data from neurons in the brain is complicated by the fact that these display variability to repeated presentations of the same stimulus [46]. The role of this noise is still unclear: noise can increase information transmission through stochastic resonance $[5,25,54]$ in the subthreshold regime at the single neuron level. In the suprathreshold regime, noise is only detrimental at the single neuron level but can increase

${ }^{*}$ Corresponding author. E-mail: maurice.chacron@mcgill.ca 
information transmission by a population [71]. Studies of neural systems are furthermore complicated by the fact that many neurons display intrinsic dynamics such as burst firing [33, 68, 69], oscillations $[32,28,34,45,66,72]$, correlations in the activity of many neurons or correlations between successive interspike intervals (ISIs) [7, 17, 18, 36, 37, 39, 43, 61] as reviewed in [24]. Finally, anatomical studies in several systems have shown that feedback projections from higher centers often vastly outnumber feedforward projections from the periphery [6, 31, 57, 69]. Finite conduction speeds through axons will induce delays in this input and can have important consequences on information processing by neurons [12, 20, 21, 26, 34, 41, 45]. Understanding the interaction between intrinsic and network induced dynamics and their role in regulating information transmission by neural assemblies is therefore of critical interest.

Electrophysiological experiments have shown that many neurons displayed a single negative ISI correlation at lag one [7, 17, 24, 36, 37, 39, 43, 61] implying that ISIs shorter than average are followed by ISIs longer than average and vice-versa. Other studies have shown that some neurons can display temporal anti-correlations in which the ISI correlation coefficients alternate in sign and decay over long lags [2]. Finally, many neurons display increased variability at very long time scales (e.g. minutes) [9, 43, 52] which are caused by weak positive ISI correlations that decay over many lags [52]. We do not consider these correlations here because their effects are felt at time scales that are often much greater than those seen in animal behavior [55]. It has been shown that both a single negative ISI correlations $[9,27,44,61]$ as well as temporal anti-correlations [23] can enhance signal detection and information transmission in isolated single neurons. In the case of a single negative ISI correlation at lag one, this process occurs through noise shaping [8, $9,10,11,40]$ : a process by which the noise power of a neurons is shifted from a certain frequency range to another and thereby increasing information transmission in the former $[56,70]$. However, noise shaping can also occur at the network level as a result of inhibitory coupling [49]. How does noise shaping occurring at the single neuron level interact with noise shaping resulting from all-toall inhibitory coupling? Recent work has shown that it was excitatory coupling, and not inhibitory coupling, that would lead to increased information transmission in networks of neurons that display intrinsic ISI correlations [1]. However, this previous study assumed that the interactions occurred instantaneously and did not include temporal delays.

Here we explore and compare the effects of adding delayed all-to-all coupling in networks composed of neurons with and without intrinsic ISI correlations. We note that such network geometry has been previously used with success to reproduce experimental results $[12,20,21]$. Further, anatomical studies have shown that the number of neurons in the brain that are connected in such a manner can range from a few to many $[47,48]$. We thus believe that our approach is realistic and can thus give results that are relevant to experimentalists.

The paper is organized as follows. The models are presented in section 2. Based on linear response theory [63], section 3 shows the theoretical calculations for information transmission [62] in neural networks with delayed feedback [12]. Section 4 shows the results, where we explore the effects of varying network size, delay time and network connectivity on the coherence and mutual information rate. We finally discuss the implications of our results in section 5 . 


\section{The models}

\subsection{Renewal and nonrenewal models}

As before, we use two neuron models: one that displays intrinsic ISI correlations (and therefore noise shaping) and one that does not $[1,10,40]$. The neuron model that displays intrinsic ISI correlations is referred as nonrenewal and the one that does not is referred to as renewal. Both are perfect integrate-and-fire models [30] where the observable output $v$ (i.e. the membrane voltage) is given by $[10,40]$ :

$$
\dot{v}=\mu+s(t)
$$

where $\mu$ is a positive bias current and $s(t)$ is a time-dependent stimulus. Every time the voltage $v$ reaches a threshold $\theta$ it is said that an action potential has occurred and $v$ is is reset to a value $\theta_{R}$. A new threshold value is then drawn uniformly within the interval $\left[\theta_{0}-D, \theta_{0}+D\right]$ thereby introducing variability in the firing sequence if $D>0$.
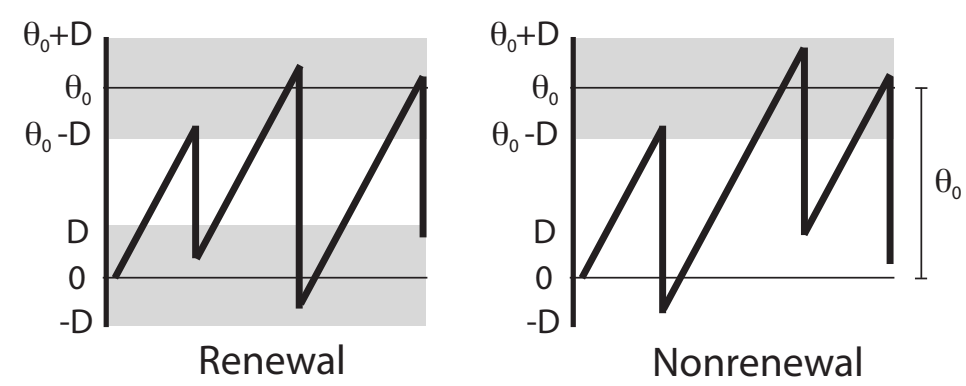

Figure 1: Example time series from the renewal (left) and nonrenewal (right) integrate and fire models. In both models, the threshold value between two consecutive firings is drawn uniformly within the interval $\left[\theta_{0}-D, \theta_{0}+D\right]$. The difference is in the reset rule whenever $v$ reaches $\theta$ : for the renewal model, the reset value of the voltage immediately after an action potential is drawn uniformly within the interval $[-D, D]$. In contrast, the voltage $v$ is decremented by a fixed amount $\theta_{0}$ in the nonrenewal model.

The difference between the two models relies on the reset rule: in the nonrenewal model, we have $\theta_{R}=\theta-\theta_{0}$ and thus $\theta_{R} \in[-D, D]$ (fig. 1). This reset rule introduces a correlation between the threshold value $\theta$ and its subsequent reset value $\theta_{R}$, thereby negatively correlating successive interspike intervals (ISI) in the absence of a time-dependent signal $s(t)[10,40]$. On the other hand, in the renewal model, $\theta_{R}$ is drawn uniformly within the interval $[-D, D]$. This makes the threshold $\theta$ and the reset value $\theta_{R}$ independent and, as a consequence, consecutive ISIs will not be correlated $[10,40]$. Both the renewal and the nonrenewal models share the same first order statistics of threshold and reset values and therefore will share the same first order ISI statistics $[10,40]$. In particular, the mean ISI is given by $\langle I\rangle=\theta_{0} / \mu$. The mean firing rate $r_{0}=1 /\langle I\rangle$ is then the same for both models and given by:

$$
r_{0}=\frac{\mu}{\theta_{0}}
$$




\subsection{Network architecture}

The $N$ model neurons are then coupled to each other through their spiking activity, in a way such that whenever any neuron fires an action potential all the neurons within the network will receive a post synaptic current after a time delay $\tau_{d} \geq 0$ (fig. 2). The activity of neuron $k$ in a given network is then given by:

$$
\dot{v}_{k}=\mu_{k}+s(t)+\frac{1}{N} \sum_{j=1}^{N} \sum_{m=1}^{M_{j}(t)} K_{k j} \gamma\left(t-t_{j}^{m}-\tau_{d}\right)
$$

where $\mu_{k}$ is the constant bias current to neuron $k, t_{j}^{m}$ is the $m^{t h}$ spike of neuron $j, \tau_{d}$ is the delay and $M_{j}(t)$ is the spike count of neuron $j$ at time $t$ (i.e. the total number of action potentials fired by neuron $j$ at time t). $K_{k j}$ represents the coupling strength between neurons $j$ and $k$ and $\gamma(t)$ is the post-synaptic current waveform given by $[1,12]$ :

$$
\gamma(t)=\Theta(t) e^{-t / \tau_{s}}
$$

where $\Theta(t)$ is the Heaviside function $(\Theta(t)=1$ if $t \geq 0 ; \Theta(t)=0$ if $t<0)$ and $\tau_{s}$ determines the rate of decay of the post-synaptic current. Throughout this study we will consider homogeneous networks and take $K_{k j}=K$ and $\mu_{k}=\mu$.

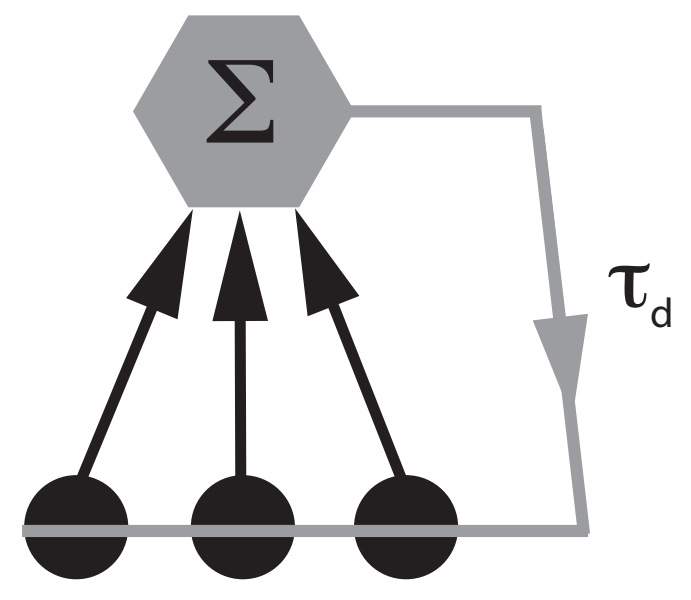

Figure 2: Network architecture. Each neuron projects to an integrator neuron which in turn feeds back to all the neurons with delay $\tau_{d}$. Note that, mathematically, this is equivalent to all-to-all coupling with a delay [21, 41]. We further note that anatomical studies have shown that such a geometry occurs in the brain and that the number of neurons that actually project to the integrator can vary from a few to many $[47,48]$. 


\section{Theory of signal processing by networks of renewal and non- renewal neurons}

We now present information theory which relies on computing the mutual information rate between the stimulus $s(t)$ and the spike train activity of either a single neuron $k$ in the network, $x_{k}(t)$, or the average spike train activity of all the neurons in the network $X(t)=\frac{1}{N} \sum_{k=1}^{N} x_{k}(t)$. We first introduce information theory and then present calculations based on linear response theory that will give an approximate expression for the mutual information rate that is valid if both the stimulus $s(t)$ and the fluctuations in the average network activity $\frac{1}{N} \sum_{k=1}^{N} x_{k}(t)$ are weak.

Throughout this study, we assume that the spike train activity of neuron $k$ is represented by a sum of Dirac delta functions centered on the spiking times $\left\{t_{k}^{j}\right\}_{j=1}^{M_{k}(t)}$ :

$$
x_{k}(t)=\sum_{j=1}^{M_{k}(t)} \delta\left(t-t_{k}^{j}\right)
$$

where $M_{k}(t)$ is the spike count of neuron $k$ at time $t$. We also take the stimulus $s(t)$ to be a zero mean Gaussian white noise with spectral height $\alpha$ that is lowpass filtered by a fourth-order Butterworth filter with cutoff frequency $f_{c}$. We note that the stimulus $s(t)$ is not correlated with the sequence of threshold and/or reset values used in each model. Therefore, the different threshold and reset values is considered as a source of noise that introduces variability in the responses of both models. The strength of this noise is controlled by the parameter $D \geq 0$ [1].

\subsection{Information theory}

Information theory was developed by Shannon $[19,67]$ in the context of telecommunications and has been widely applied in neuroscience $[3,8,12,13,14,15,16,17,18,38,50,58,62,65]$. If the stimulus $s(t)$ has a Gaussian probability distribution, then a lower bound on the mutual information rate $\mathrm{MI}$ is given by $[3,62]$ :

$$
M I=\int_{0}^{f_{c}} I(f) d f
$$

Note that the mutual information rate is expressed in bits/(unit time) and quantifies the system's ability to distinguish between different stimuli: $X$ bits/(unit time) implies that $2^{X}$ stimuli can be distinguished by looking at the activity over one time unit $[3,19]$. As time is dimensionless in our study, the mutual information rate $M I$ is thus expressed in bits. Here $I(f)$ is the mutual information rate density given by:

$$
I(f)=-\log _{2}[1-C(f)]
$$

and $C(f)$ is the coherence function given by:

$$
\left.C_{(} f\right)=\frac{\left|P_{x s}(f)\right|^{2}}{P_{x x}(f) P_{s s}(f)}
$$


where $P_{y y}(f)=\left\langle|\hat{x}(f)|^{2}\right\rangle$ is the power spectrum of the neuronal response $x(t)$ to the stimulus $s(t)$ with $\hat{x}(f)$ the Fourier transform of $x(t), P_{s s}(f)=\left\langle\left|\hat{s}_{t}(f)\right|^{2}\right\rangle$ is the power spectrum of the stimulus $s(t)$ with $\hat{s}_{t}(f)$ the Fourier transform of $s(t)$, and $P_{x s}(f)=\left\langle\hat{x}^{*}(f) \hat{s}_{t}(f)\right\rangle$ is the cross spectrum between $x(t)$ and $s(t)$, where $\hat{x}^{*}(f)$ denotes the complex conjugate of $\hat{x}(f)$. Here the $\langle\ldots\rangle$ denotes averaging of realizations. We note that the lower bound given by equation (3.2) is equal to the actual mutual information rate in the case of a linear system [3, 64].

\subsection{Linear response theory}

According to linear response theory, the activity of a neuron in the network in the presence of a time-varying stimulus can be approximated by the baseline activity (i.e. $s(t)=0$ and $K=0$ ) of an isolated neuron, $x_{0}(t)$, plus a small perturbation [63]. Linear response theory has been applied before to describe the behavior of neurons in response to weak input $[1,10,12,21,40,41]$.

The spike train of a single neuron $k$ in response to a small time dependent perturbation $A_{k}(t)$ is then given by:

$$
x_{k}(t)=x_{0 k}(t)+\left(\chi_{k} * A_{k}\right)(t),
$$

were $\chi_{k}(t)$ is the susceptibility of neuron $k$ in response to the input. The "** operation represents a convolution. We note that for a stochastic linear system, equation (3.5) is usually $\left\langle x_{k}(t)\right\rangle=\left\langle x_{0 k}(t)\right\rangle+\left(\chi_{k} * A_{k}\right)(t)$, where $\langle\ldots\rangle$ denotes an average over realizations. However, equation (3.5) will hold approximately for large $D[1,21,40,41]$. As before [1, 12, 21, 41], we take the perturbation $A_{k}(t)$ to be composed of the common time varying stimulus as well as the average network activity received by neuron $k$ :

$$
A_{k}(t) \equiv A(t)=s(t)+\frac{1}{N} \sum_{j=1}^{N} \sum_{m=1}^{M_{j}(t)} K \gamma\left(t-t_{j}^{m}-\tau_{d}\right)
$$

Taking the Fourier transform of equation (3.5) gives:

$$
\hat{x}_{k}(f)=\hat{x}_{0 k}(f)+\hat{\chi}_{k}(f) \hat{A}(f)
$$

where $\hat{x}_{k}(f), \hat{A}(f), \hat{x}_{0 k}(f)$, and $\hat{\chi}_{k}(f)$ are the Fourier transforms of $x_{k}(t), A(t), x_{0 k}(t)$, and $\chi_{k}(t)$, respectively. Applying equation (3.6) into equation (3.5) and taking the Fourier transform on both sides yields:

$$
\begin{aligned}
& \hat{x}_{k}(f)=\hat{x}_{0 k}(f)+\hat{\chi}_{k}(f) \hat{s}_{t}(f)+\hat{\phi}_{k}(f) \frac{1}{N} \sum_{n=1}^{N} \hat{x_{n}}(f) \\
& \hat{\phi}_{k}(f)=K \hat{\chi}_{k}(f) \hat{\gamma}(f) \exp \left(-2 \pi i f \tau_{d}\right)
\end{aligned}
$$

where $\hat{s}_{t}(f)$ is the Fourier transform of $s(t)$ and $\hat{\gamma}(f)$ is the Fourier transform of $\gamma(t)$ given by: 


$$
\hat{\gamma}(f)=\frac{1}{1 / \tau_{s}+i 2 \pi f}
$$

Previous studies have found that the susceptibilities of both the renewal and the nonrenewal models are equal and given by $[10,40]$ :

$$
\hat{\chi}_{k}(f) \equiv \hat{\chi}(f)=\frac{1}{\theta_{0}}
$$

we thus have:

$$
\hat{\phi}_{k}(f) \equiv \hat{\phi}(f)=K \hat{\chi}(f) \hat{\gamma}(f) \exp \left(-2 \pi i f \tau_{d}\right)
$$

\subsubsection{Mutual Information rate density between the average network activity and the stim- ulus}

In this section, we derive an expression for the coherence between the average network activity and the stimulus $s(t)$. The average network activity can be found by summing equation (3.8) from $k=1$ to $N$ and dividing by $N$. After rearranging terms we obtain:

$$
\frac{1}{N} \sum_{k=1}^{N} \hat{x}_{k}(f)=\frac{\frac{1}{N} \sum_{k=1}^{N} \hat{x}_{0 k}+\hat{\chi}(f) \hat{s}_{t}(f)}{1-\hat{\phi}(f)}
$$

From this we can find the average network activity spectrum. If we set $\hat{X}(f)=\frac{1}{N} \sum_{k=1}^{N} \hat{x}_{k}(f)$ and denote its complex conjugate by $\hat{X}(f)^{\star}$, then the power spectrum of the average activity $P_{X X}(f)=\left\langle\hat{X}(f) \hat{X}(f)^{\star}\right\rangle$, where the $\langle\ldots\rangle$ denotes an average over realizations, is given by:

$$
P_{X X}(f)=\left|\frac{1}{1-\hat{\phi}(f)}\right|^{2}\left[\frac{1}{N} P_{00}(f)+|\hat{\chi}(f)|^{2} P_{s s}(f)\right]
$$

Here $P_{00}(f)$ is the power spectrum of a single neuron in the absence of coupling and stimulation. Expressions have already been derived for the power spectra $P_{00 r}(f)$ and $P_{00 n}(f)$ of renewal and nonrenewal model single neurons, respectively [10, 40]:

$$
\begin{aligned}
P_{00 r}(f)= & r_{0}\left[(\beta f)^{4}-\sin ^{4}(\beta f)\right] / \Gamma \\
\Gamma= & (\beta f)^{4}- \\
& 2(\beta f)^{2} \sin ^{2}(\beta f) \cos \left(2 \pi f / r_{0}\right)+ \\
& \sin ^{4}(\beta f) \\
P_{00 n}(f)= & r_{0}- \\
& \frac{\sin ^{2}(\beta f)}{(\beta f)^{2}}\left(r_{0}-r_{0}^{2} \sum_{n=-\infty}^{\infty} \delta\left(f-n r_{0}\right)\right)
\end{aligned}
$$


where $\beta=\frac{2 \pi D}{\mu}$, and $r_{0}$ is the baseline firing rate given by equation (2.2).

The cross-spectrum, $P_{X s}(f)$, between the average network activity $X(t)$ and the time-varying stimulus $s(t)$ is obtained by evaluating $P_{X s}(f)=\left\langle\hat{X}(f) \hat{s}_{t}(f)^{\star}\right\rangle$, where $\hat{s}_{t}(f)^{\star}$ is the complex conjugate of $\hat{s}_{t}(f)$. This yields:

$$
P_{X s}(f)=\frac{\hat{\chi}(f)}{1-\hat{\phi}(f)} P_{s s}(f)
$$

where $P_{s s}(f)$ is the power spectrum of the time-varying stimulus. Finally, the coherence between the average network activity and the stimulus can be obtained by equation (3.4):

$$
C_{X s}(f) \equiv C_{n e t w o r k}(f)=\frac{|\hat{\chi}(f)|^{2} P_{s s}(f)}{\frac{1}{N} P_{00}(f)+|\hat{\chi}(f)|^{2} P_{s s}(f)}
$$

The mutual information rate can then be obtained from equation (3.18) using equations (3.2) and (3.3). Note that the expression for the coherence between the average network activity and the time-varying stimulus given by equation (3.18) does not depend on the term $\hat{\phi}(f)$ and therefore it does not depend directly on the coupling strength $K$ and the delay $\tau_{d}$ [1]. Instead, the dependence of the coherence on the coupling comes indirectly through the dependence of $P_{00}(f)$ on the bias current $\mu$. We note that this does not mean that the delay does not affect the firing dynamics as both power spectrum given by equation (3.14) and the cross-spectrum given by equation (3.17) of the average network activity both strongly depend on the delay $\tau_{d}$ through $\hat{\phi}(f)$ and this effect has been seen previously $[12,42]$. It has been shown in previous studies that in a network of LIF neurons the input to each neuron can be decomposed into a constant and time-dependent components $[12,21,41]$. The new bias current $\mu^{\prime}$ is composed of the bias current $\mu$ in the absence of coupling plus a contribution from the average network activity $[1,12,21,41]$ :

$$
\mu^{\prime}=\mu+K \tau_{s} r_{0}\left(\mu^{\prime}\right)
$$

where $r_{0}\left(\mu^{\prime}\right)$ is the firing rate of a single uncoupled integrate-and fire neuron with bias $\mu^{\prime}$. Substituting $r_{0}\left(\mu^{\prime}\right)=\mu^{\prime} / \theta_{0}$ in equation (3.19) and solving for $\mu^{\prime}$ gives [1]:

$$
\mu^{\prime}=\frac{\mu}{1-\frac{K \tau_{s}}{\theta_{0}}}
$$

Thus, while the average network activity depends on the coupling strength $K$, it does not depend on the delay $\tau_{d}$. The situation is however different if one computes the information rate from a single neuron within the network.

\subsubsection{Mutual Information rate density between a single neuron within the network and the stimulus}

In this section, we derive an expression for the coherence between the spike train of a single neuron $x_{k}(t)$ and the stimulus $s(t)$. An expression for the power spectrum of $x_{k}(t)$ can be found by substituting equation (3.13) into equation (3.8): 


$$
\hat{x}_{k}(f)=\hat{x}_{0 k}(f)+\hat{\chi}(f) \hat{s}_{t}(f)+\hat{\phi}(f) \frac{\frac{1}{N} \sum_{n=1}^{N} \hat{x}_{0 n}(f)+\hat{\chi}(f) \hat{s}_{t}(f)}{1-\hat{\phi}(f)}
$$

The power spectrum of the output spike train of neuron $k$ can be obtained by computing $P_{x_{k} x_{k}}(f)=\left\langle\hat{x_{k}}(f)^{\star} \hat{x_{k}}(f)\right\rangle$, which gives:

$$
P_{x_{k} x_{k}}(f)=P_{00}(f)\left\{\frac{N-1}{N}+\frac{1}{N}\left|\frac{1}{1-\hat{\phi}(f)}\right|^{2}\right\}+|\hat{\chi}(f)|^{2} P_{s s}(f)\left|\frac{1}{1-\hat{\phi}(f)}\right|^{2}
$$

We next have to evaluate the cross-spectrum between neuron $k$ and the stimulus given by $P_{x_{k} s}(f)=\left\langle\hat{x}_{k}(f) \hat{s}_{t}(f)^{\star}\right\rangle$, which yields:

$$
P_{x_{k} s}(f)=\frac{\hat{\chi}(f)}{1-\hat{\phi}(f)} P_{s s}(f)
$$

we note that the expression for $P_{x_{k} x_{k}}(f)$ given by equation (3.22) and the expression for $P_{x_{k} s}$ given by equation (3.23) do not depend on $k$. Thus, all neurons in the network will have the same power spectra and cross-spectra and will thus have the same coherence $C_{x_{k} s}(f) \equiv C(f)$ which can be obtained by substituting equations (3.22) as well as (3.23) into equation (3.4) [12]:

$$
C(f)=\frac{|\hat{\chi}(f)|^{2} P_{s s}(f)\left|\frac{1}{1-\hat{\phi}(f)}\right|^{2}}{|\hat{\chi}(f)|^{2} P_{s s}(f)\left|\frac{1}{1-\hat{\phi}(f)}\right|^{2}+P_{00}(f)\left\{\frac{N-1}{N}+\frac{1}{N}\left|\frac{1}{1-\hat{\phi}(f)}\right|^{2}\right\}}
$$

The mutual information rate can then be obtained from equation (3.24) using equations (3.2) and (3.3). It is worth noting that when the network is composed of one neuron (i.e. $N=1$ ), we have $C(f)=C_{\text {network }}(f)$ and $C(f)$ furthermore does not depend on the delay $\tau_{d}$.

We note that the only difference between the coherence of single renewal and nonrenewal neurons and the stimulus in a homogeneous network comes from the baseline powerspectrum $P_{00}(f)$. Equations (3.15) or (3.16) can be substituted into equation (3.24) in order to obtain the coherence between single renewal or nonrenewal neurons and the stimulus, respectively.

\section{Noise shaping by global delayed feedback}

It was previously shown that suitable scaling of the noise parameter $D$ would lead to similar mutual information rates in both models [1]. Thus, in order to better compare the effects of the delay on the two models we chose the noise intensities $D_{n}$ and $D_{r}$ for the nonrenewal and renewal model neurons, respectively, such that both models would have the same mutual information rates. 


\subsection{Network size}

We first examined the dependence of the coherence of a single neuron in a network $C(f)$ on the network size $N$. Figure 3 shows the coherence $C(f)$ for networks of renewal (figures $3 \mathrm{a}$ and $3 \mathrm{~b}$ ) and nonrenewal (figures $3 \mathrm{c}$ and $3 \mathrm{~d}$ ) models as a function of frequency for different network sizes $N$. The simulations are shown as symbols and the theoretical result, given by equation (3.24), are shown as a continuous line. Note that we have $C(f)=1$ of the nonrenewal model at $f=0$, this is due to the fact that the power spectrum $P_{00 n}(0)=0$ for this model $[10,40]$. For the renewal model, we have $P_{00 r}(0)=2 D^{2} \mu /\left(3 \theta_{0}^{3}\right)>0$ and thus $C(f)<1$ [1]. When $N=1$ there is no resonance in the coherence due to the fact that it doesn't depend on $\phi(f)$ which can be seen by substituting $N=1$ in equation (3.24). For $N>1$ a resonance appears at approximately the inverse of the delay $\tau_{d}$ for positive coupling (i.e. $K>0$ ) and at the inverse of $2 \tau_{d}$ for negative coupling (i.e. $K<0$ ). In both models the resonances in the coherence $C(f)$ become more prominent as the network size increases but converge to limit values as $N \rightarrow \infty$. It can be seen that the coherence value at given frequencies saturates as $N$ increases in figures $3 \mathrm{e}$ and $3 \mathrm{f}$ for renewal and nonrenewal networks, respectively.

It was previously shown in [12] that the coherence increases at the resonant frequency as the network size increases and that this effect is due to a decrease in the power spectrum of a single neuron $P_{x x}(f)$, while the cross-spectrum $P_{x s}(f)$ remains constant. Since the coherence $C(f)$ is proportional to the ratio $\left|P_{x s}(f)\right|^{2} / P_{x x}(f)$ for fixed $f$, the coherence $C(f)$ increases whenever $P_{x x}(f)$ decreases and $P_{x s}(f)$ remains constant [12]. Intuitively, this occurs because, as $\mathrm{N}$ increases, the variance of the average network activity decreases and therefore contributes less power to the power spectrum of an individual neuron. The difference between excitatory and inhibitory coupling can be understood as follows. For excitatory coupling, the single neuron will tend to oscillate with a period equal to the delay $\tau_{d}$. However, for inhibitory coupling, the single neuron will tend to oscillate with a period equal to twice the delay $\tau_{d}$ : the neuron fires at $t=0$, it is then inhibited around $t=\tau_{d}$, and the release from inhibition causes another firing near $t=2 \tau_{d}$ $[12,21,41]$. These oscillations translate to peaks in the power spectrum at integer multiples of $1 / \tau_{d}$ for excitatory coupling and $0.5 / \tau_{d}$ for inhibitory coupling [12].

Adding a delay will thus differentially affect the mutual information rate $M I$ depending on the frequency content of the stimulus $s(t)$. For excitatory coupling, adding a delay will increase the coherence (and thus the mutual information rate density) in the frequency ranges $\left[0,0.5 \tau_{d}^{-1}\right]$ and $\left[(0.5 n-0.25) \tau_{d}^{-1},(0.5 n+0.25) \tau_{d}^{-1}\right]$ for integer $n>0$ even. In contrast, the coherence will decrease in the frequency ranges $\left[(0.5 n-0.25) \tau_{d}^{-1},(0.5 n+0.25) \tau_{d}^{-1}\right]$ for integer $n>0$ odd. In contrast, adding a delay with inhibitory coupling will increase the coherence in the frequency ranges $\left[(0.25 n-0.125) \tau_{d}^{-1},(0.25 n+0.125) \tau_{d}^{-1}\right]$ for integer $n>0$ odd and decrease the coherence in the frequency ranges $\left[0,0.25 \tau_{d}^{-1}\right]$ and $\left[(0.25 n-0.125) \tau_{d}^{-1},(0.25 n+0.125) \tau_{d}^{-1}\right]$ for integer $n>0$ even.

Figure 4 shows the mutual information rate $M I$ as a function of network size, $N$ for both models. For the renewal model, we chose $f_{c}=6$ and $\tau_{d}=0.01$ such that increasing $N$ increases the coherence over the frequency range $[0,6]$ (figure $4 \mathrm{a}$, inset). For the nonrenewal model, we chose $f_{c}=20$ and $\tau_{d}=0.025$ such that increasing $N$ also increases the coherence over the frequency 

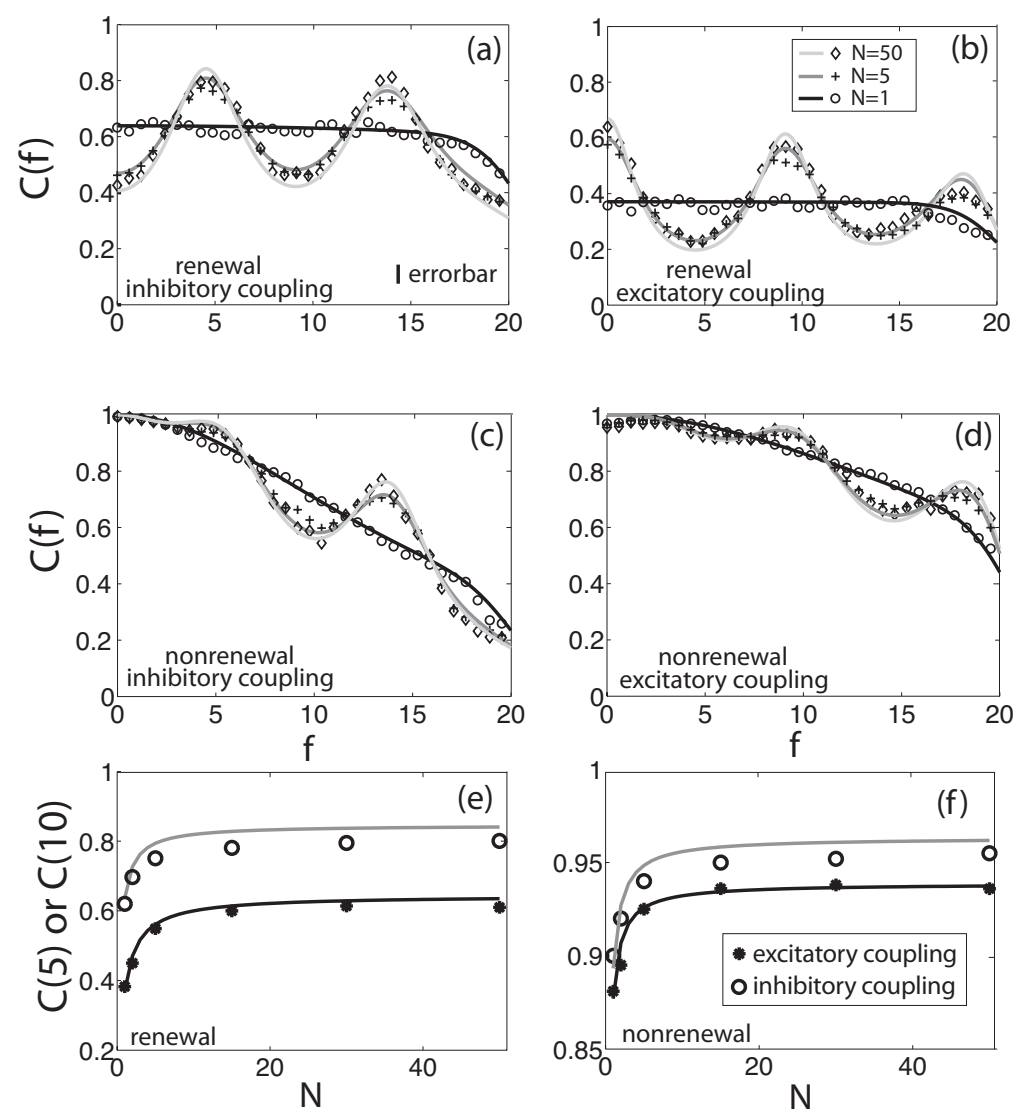

Figure 3: Coherence $C(f)$ between a single neuron and the stimulus as a function of frequency $f$ for different network sizes, $N$. (a) Coherence for a network composed of renewal neurons with inhibitory coupling in a network of 1 (circle, black), 5 (cross, dark gray), and 50 (diamond, light gray) neurons. The symbols are the numerical simulations and the solid lines are obtained from the theory. (b) Coherence for the same network but with excitatory coupling. (c) Coherence for a network composed of nonrenewal neurons with inhibitory coupling. (d) Coherence for a network composed of nonrenewal neurons with excitatory coupling. (e) Coherence value at $f=$ 10 for excitatory coupling (solid circles) and coherence value at $f=5$ for inhibitory coupling (hollow circles) as a function of the network size $N$ for a network composed of renewal neurons. Theoretical values are shown as solid lines for both cases and agree very well with numerical simulations. (f) Coherence value at $f=10$ for excitatory coupling (solid circles) and coherence value at $f=5$ for inhibitory coupling (hollow circles) as a function of the network size $N$ for a network composed of nonrenewal neurons. Theoretical values are shown as solid lines for both cases and agree very well with numerical simulations. Parameter values used for all the simulations were $\theta_{0}=2, \mu=300, \tau_{d}=0.1, \tau_{s}=0.01, f_{c}=20, \sigma=27, D_{n}=1, D_{r}=0.4$, and $K=-100$ (inhibitory coupling) or 100 (excitatory coupling). In this and subsequent figures, the errorbar gives the standard error from 10 numerical simulations of the networks. 

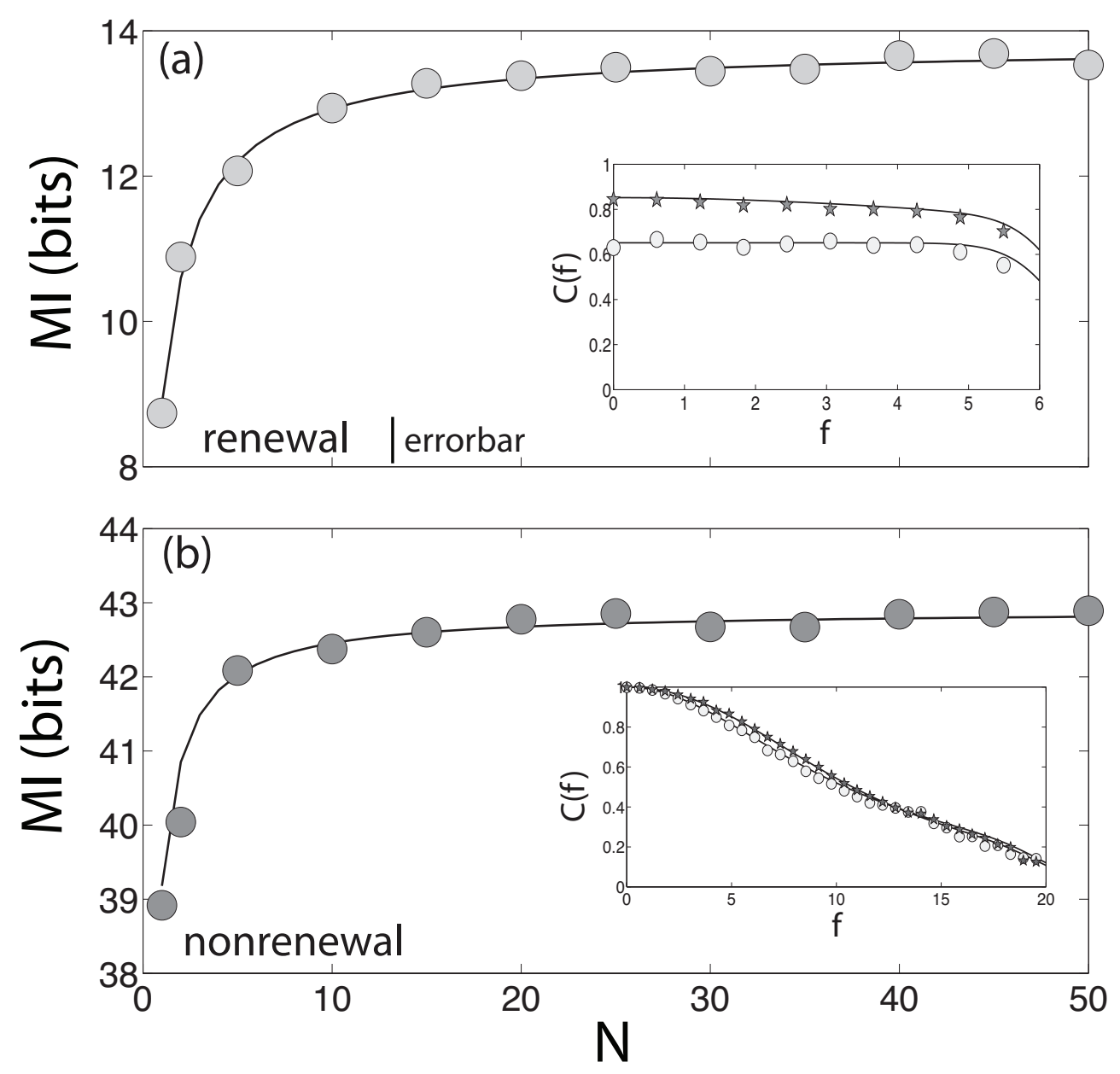

Figure 4: Mutual information rate $M I$ transmitted by a single neuron as a function of network size $N$ for a network composed of renewal (a) and nonrenewal (b) model neurons. The numerical simulations are shown as symbols while the theory is shown as a solid line. In (a), parameters are the same as in fig. 3 except $\tau_{d}=0.01$ and $f_{c}=6$. The inset shows the coherence for $N=1$ (circles) and $N=10$ (stars) for these parameters. In (b), parameters are the same as in fig. 3 except $\tau_{d}=0.025$. The inset shows the coherence for $N=1$ (circles) and $N=10$ (stars) for these parameters. 
range $[0,20]$ (figure $4 \mathrm{~b}$, inset). It can be seen that the mutual information rate $M I$ increases as a function of $N$ and saturates for large $N$. Figure 4 shows that for both models the biggest change in $M I$ happens when the network is composed from $N=1$ to $N=10$. It can be seen that the qualitative behavior of both graphs is essentially the same although the nonrenewal model has a larger mutual information rate $M I$ due to the fact that the coherence is unity for $f=0$.

We conclude that adding a delay has qualitatively the same effect in both models and will create resonances in the coherence in frequency ranges that will depend on the sign of the coupling strength $K$. The differences observed for both models are largely due to the differences in their coherence curves with $K=0$ that have been previously explained in detail [10,40]. The fact that the coherence $C(f)$ is unity for $f=0$ for the nonrenewal model will attenuate the relative effects of delayed feedback near $f=0$ since $0 \leq C(f) \leq 1$ by definition.

\subsection{Delay time}

We now explore the consequences of varying the delay $\tau_{d}$ on the resonance in the coherence $C(f)$ of both models. Figure 5 shows the coherence $C(f)$ for both renewal and nonrenewal models as a function of frequency $f$ for different delay times: $\tau_{d}=0.1$ (diamonds), 0.05 (stars) and 0.025 (crosses).

It can be seen in figure 5 that varying the delay $\tau_{d}$ changes the frequency at which the coherence $C(f)$ has a resonance for both renewal (figure 5a) and nonrenewal (figure 5b) networks. As mentioned above, inhibitory coupling is expected to create local maxima in the coherence located near integer multiples of $0.5 \tau_{d}^{-1}$ which is approximately true. The main difference with the models relies in that coherence $C(f)$ decreases as $f$ increases for the nonrenewal model (figure $5 \mathrm{~b}$ ) whereas it is approximately constant for the renewal model in the absence of feedback (figure 5a). Due to this effect, the local maxima in the coherence of the nonrenewal model neuron decrease in height with increasing $f$ (see for instance when $\tau_{d}=0.1$ ) whereas the first two local maxima in the coherence $C(f)$ created by the same delay have approximately the same height for the renewal model neuron.

An alternative way to see the effects of the feedback delay time on the information transmission is through the mutual information rate $M I$ for different values of the delay $\tau_{d}$. Figure 6 shows the mutual information rates $M I$ for both models as a function of the delay $\tau_{d}$ for a single neuron in a network composed of renewal (figure 6a) and nonrenewal (figure 6b) model neurons when they are coupled with excitation (triangles up) or inhibition (triangles down). The solid lines represent the theory for both cases and the dashed line show the value of the mutual information rate $M I$ when the networks are uncoupled. The noise level was adjusted such that both model neurons display the same mutual information rate $M I$ when uncoupled. It can be seen that, with excitatory coupling, the mutual information rate $M I$ decreases with increasing delay in both models. The situation is opposite, however, when the networks are coupled with inhibition: the $M I$ has a minimum value when $\tau_{d}=0$ from which it increases until reaching a constant value.

The main difference between both models is the value to which the mutual information rate $M I$ converges to for large delay with respect to the value for uncoupled networks. For the nonrenewal model, the mutual information rate $M I$ is always lesser/greater than its value for no coupling for 

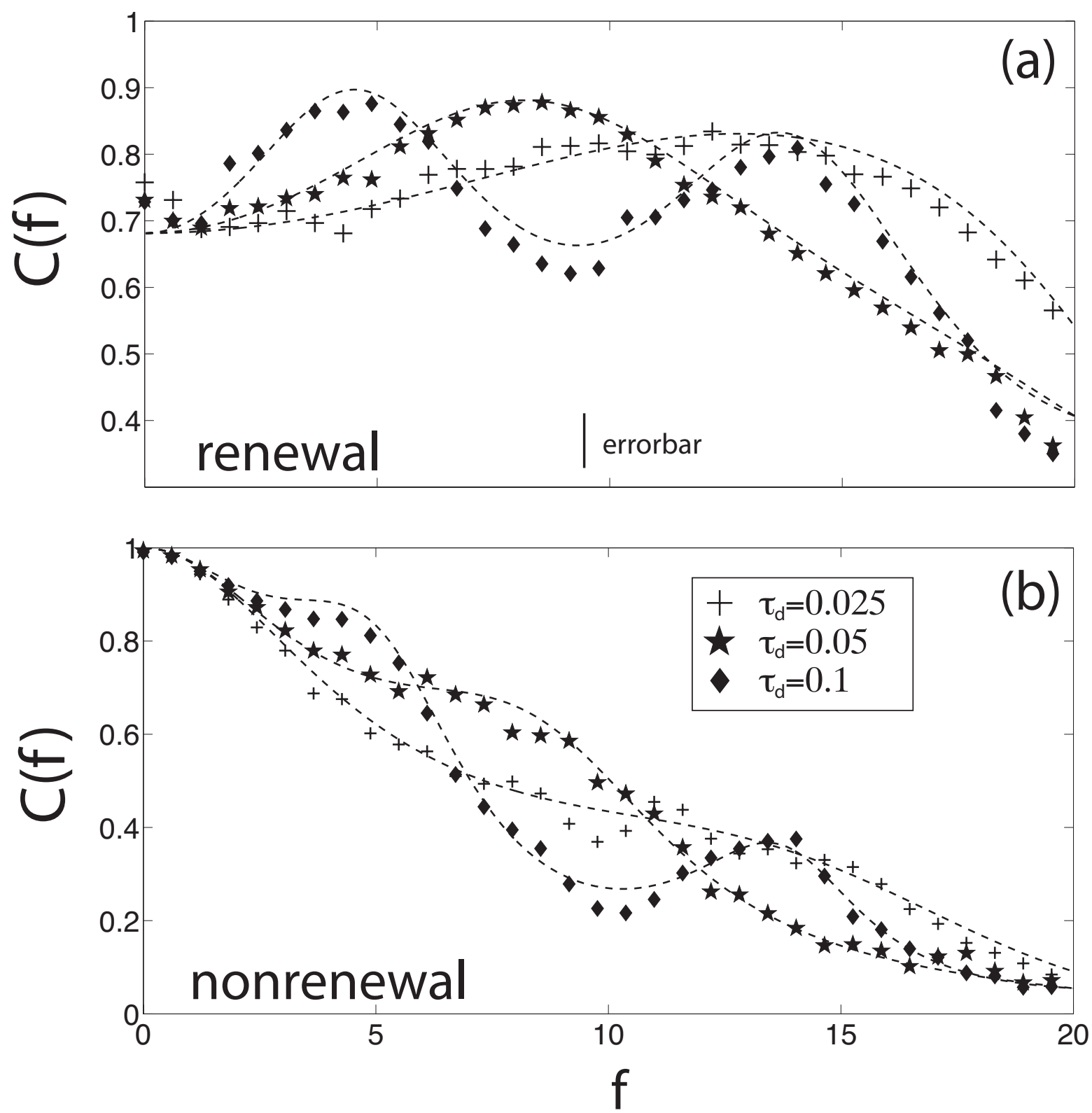

Figure 5: Coherence $C(f)$ of a single neuron in a network of renewal (a) and nonrenewal (b) neurons with inhibitory coupling for different delay times: 0.1 (diamonds), 0.05 (stars), 0.025 (crosses). The symbols represent the numerical simulations and the dashed lines the theoretical results. It can be seen there is good agreement between the simulations and the theory. Other parameters have the same value as in fig. 3 except $\theta=5$ and $K=-100$. We also used $N=10$. The parameters used are such that the $M I$ is the same for both models when $K=0$. 

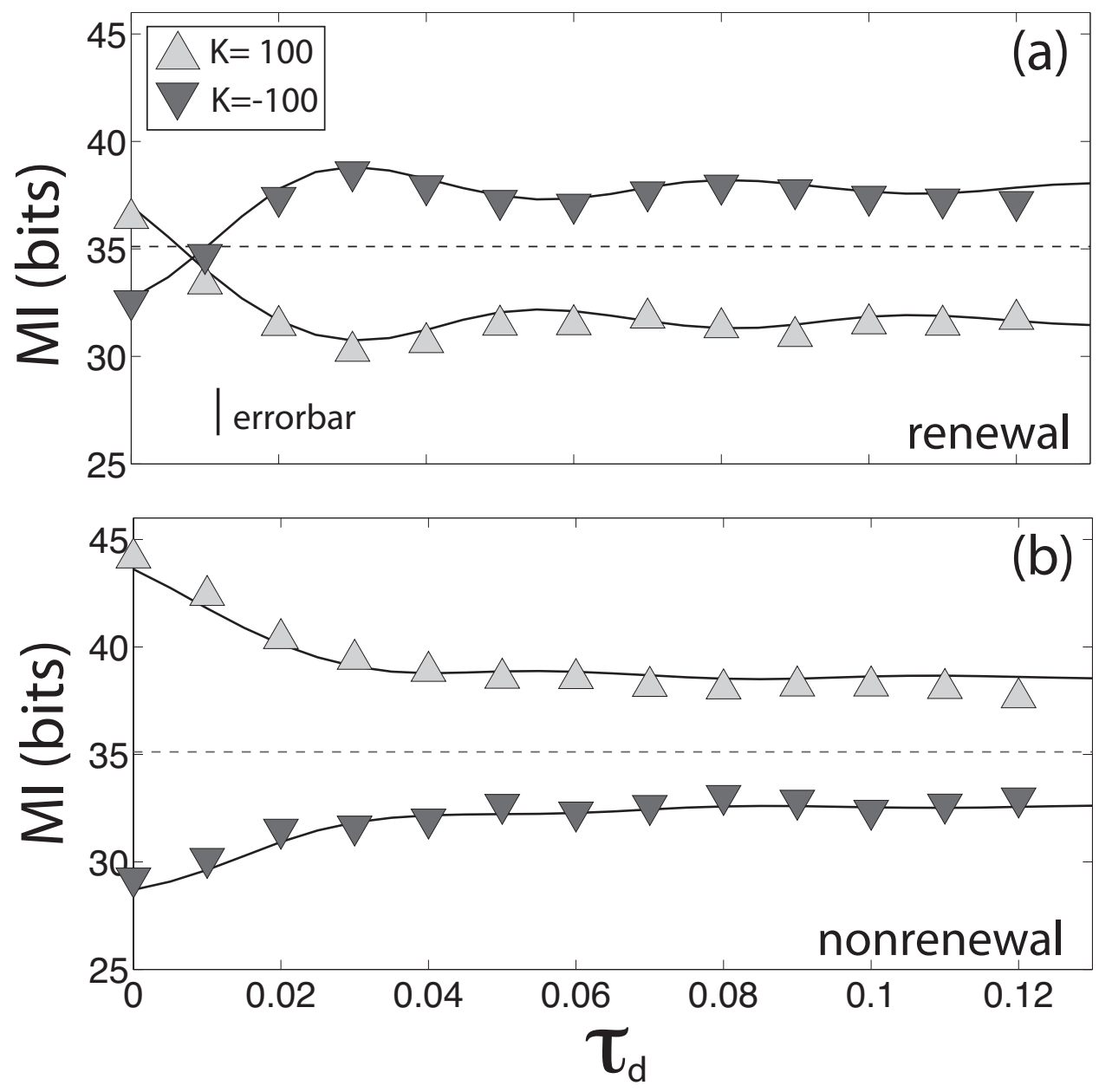

Figure 6: Mutual information rate $M I$ as a function of delay time for a neuron in a renewal network, (a), and one in a nonrenewal network (b). Symbols show the numerical simulations and the solid line the theoretical results. Triangles up represent excitatory coupling and triangles down inhibitory coupling. Dashed line represents the value of the $M I$ when the network is uncoupled. Other parameters have the same value as in figure 5. 
inhibitory/excitatory coupling for any value of the delay $\tau_{d}$. For the renewal model, the mutual information rate $M I$ increases/decreases to a value that is greater/lesser than value for no coupling for inhibitory/excitatory coupling. In order to understand why this effect happens it is necessary to explore the effects of different couplings on the coherence and $M I$.

\subsection{Network connectivity}

We finally explore the role of the network coupling strength $K$. Figure 7 shows the coherence $C(f)$ for single neurons in networks of renewal (figure 7a) and nonrenewal (figure 7b) neurons with different connectivities: inhibitory (triangles down), excitatory (triangles up) and no coupling (circles). As in the previous examples one of the differences between the models relies on the fact that the coherence for the nonrenewal model is one at $f=0$ while for the renewal model it reaches a fixed value less than one at $f=0$. It can also be seen that the resonances caused by the delayed feedback appear at the same frequencies for both models.

However, an interesting difference between both models is that compared to the situation where the networks are not connected (represented in circles), excitatory coupling lowers the coherence in the renewal model and it increases it in the nonrenewal. The situation is opposite for inhibitory coupling: the mean value of the coherence $C(f)$ over the frequency range $\left[0, f_{c}\right]$ increases compared to the uncoupled situation for the renewal model neuron and decreases for the nonrenewal model neuron.

This situation can be better visualized when computing the mutual information rate $M I$ for different coupling. Figure 8 shows the mutual information rate as a function of $K$ when the networks have no delay (represented as hollow circles) and with a delay (stars) for renewal (figure 8a) and nonrenewal (figure $8 b$ ) models. For $\tau_{d}=0$, both models show an increase in the mutual information rate $M I$ as a function of increasing coupling strength $K$. However, adding a delay $\tau_{d}=0.1$ causes the mutual information rate $M I$ to decrease with increasing coupling strength $K$ for the renewal model only (figure 8a) and not for the nonrenewal model (figure 8b). These results show that intrinsic ISI correlations can have a profound effect on how delayed feedback can alter information transmission by neural networks.

\section{Discussion}

We have explored the effects of delayed global feedback on information transmission in networks of renewal and nonrenewal neurons. Specifically, we found that adding a delay $\tau_{d}$ can cause local maxima and minima in the coherence function between a single neuron's spike train and the stimulus. The value of the delay $\tau_{d}$ as well as the sign of the coupling coefficient $K$ both determine the location of these maxima/minima. Most interestingly, we found that delays could have differential effects on the mutual information rate $M I$ transmitted by individual renewal and nonrenewal neurons embedded in a network. Specifically, we found that increasing the delay $\tau_{d}$ will increase/decrease the overall coherence for excitatory/inhibitory coupling in nonrenewal/renewal 

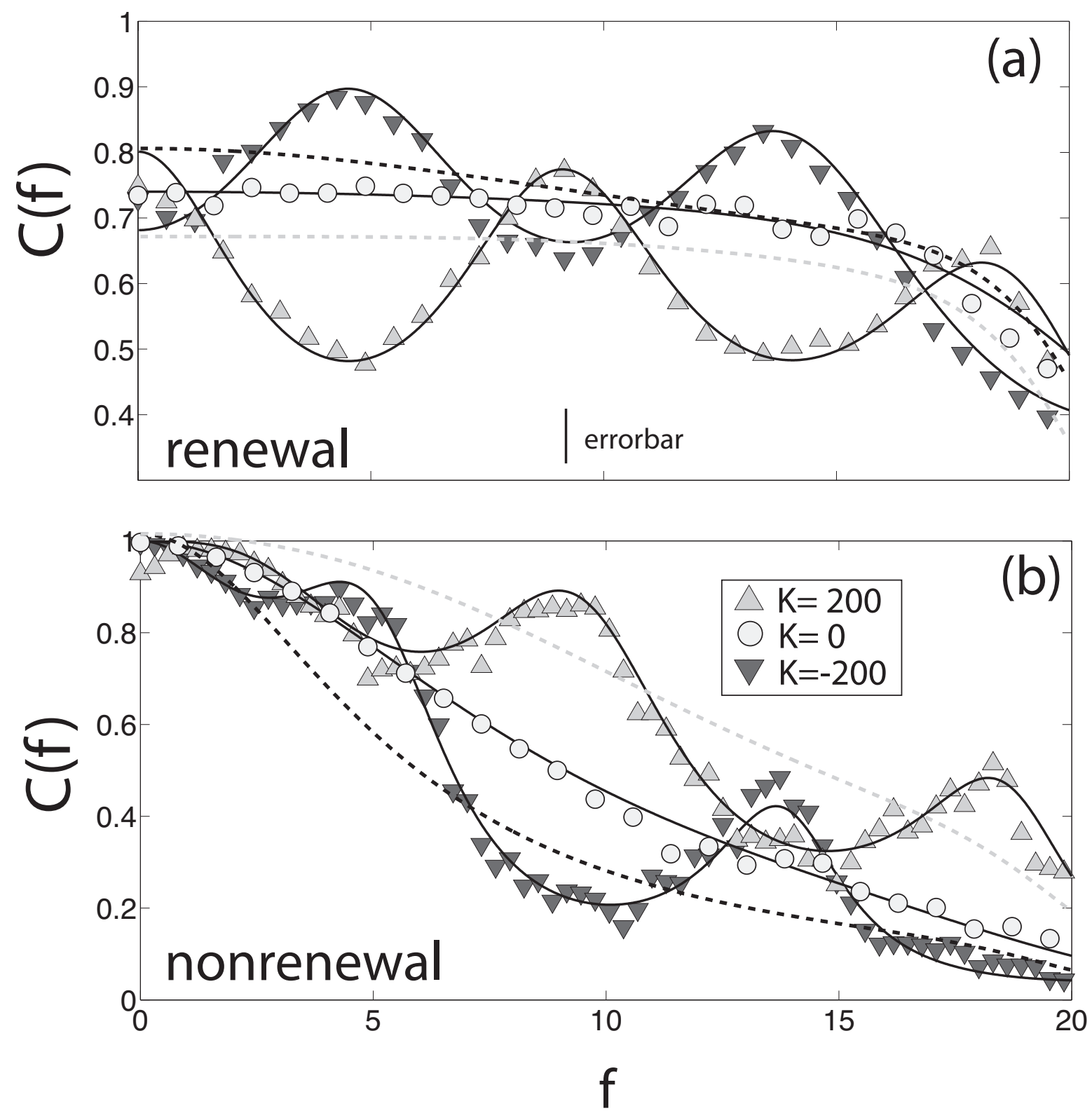

Figure 7: Coherence $C(f)$ of a single neuron in a network of renewal (a) and nonrenewal (b) neurons with a fixed delay $\tau_{d}=0.1$ for different values of the coupling strength $K$, inhibitory coupling (dark triangles down), zero coupling (circles) and excitatory coupling (light triangles up). Other parameters have the same value as in fig 5. In both (a) and (b), the dashed lines show the coherence for $\tau_{d}=0$ and inhibitory (black) and excitatory (gray) coupling. Whereas the resonance due to the coupling happens at the same frequency for both models, excitatory coupling tends to increase the coherence for the nonrenewal model as compared to zero coupling whereas the coherence decreases for the renewal model when coupled with excitation. The story is opposite for inhibitory coupling. 

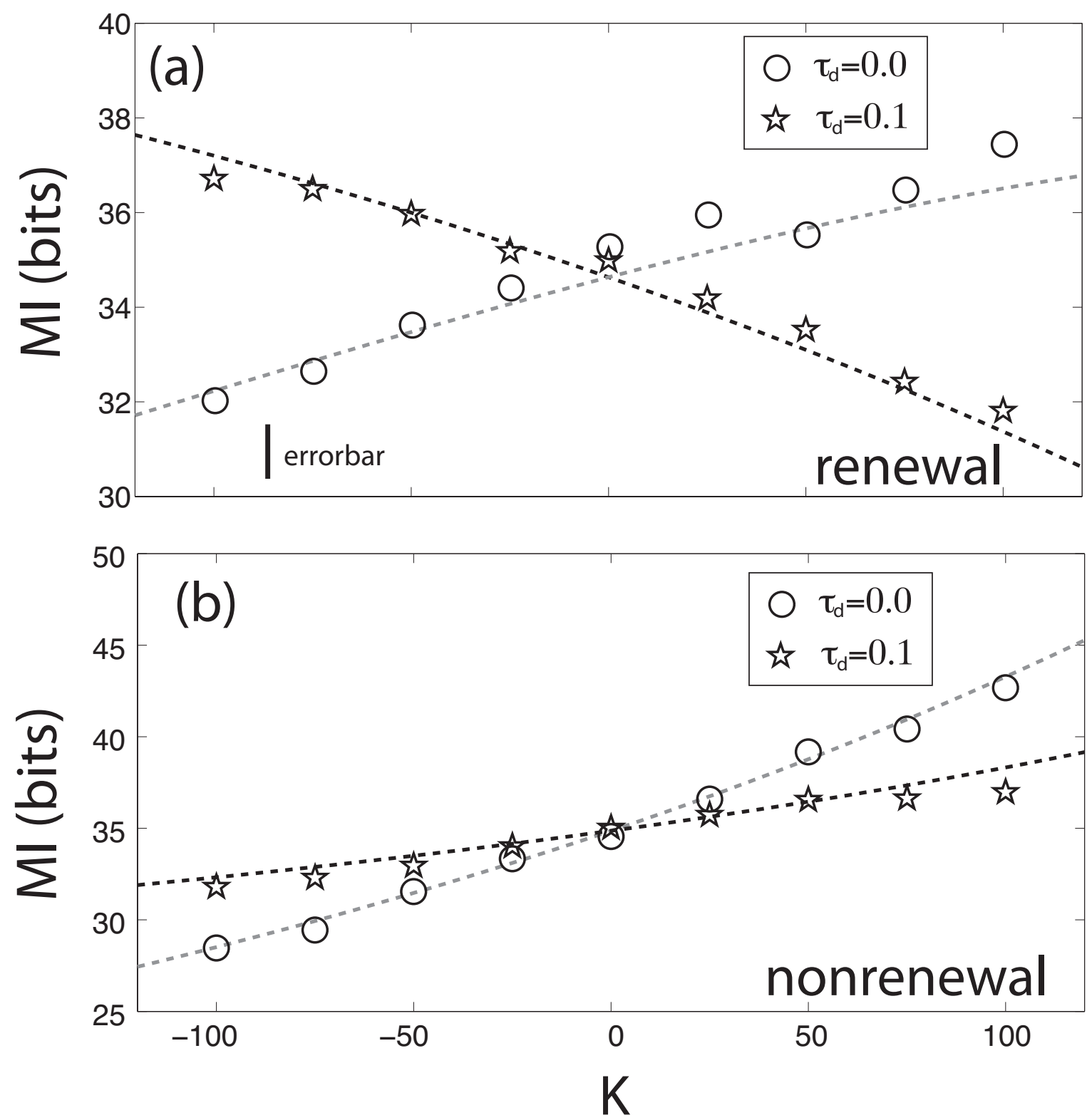

Figure 8: Effects of adding a delay on the mutual information rate $M I$ as a function of coupling strength $K$ for a neuron in a renewal network (a) and a neuron in a nonrenewal network (b) with $\tau_{d}=0$ (circles) and $\tau_{d}=0.1$ (stars). Other parameters have the same values as in fig. 5 . 
model neurons. However, we note that, for $\tau_{d}=0$, the coherence increases/decreases for excitatory/inhibitory coupling for both model neurons. Thus, for renewal neurons, the delay qualitatively changed the effect of coupling in the mutual information rate. In contrast, adding a delay did not qualitatively change the effects of coupling on the mutual information rate for nonrenewal neurons.

Our results complement those of [1] where information transmission by networks of renewal and nonrenewal neurons was investigated using methods similar to our own. However, our results focus on the activity of single neurons in the presence of delays whereas [1] only looked at the average network activity in the absence of delays. Our theoretical calculations show in fact that addition of a delay $\tau_{d}$ will surprisingly not affect information transmission by the average network activity, thereby generalizing the results found in [1]. In contrast, the delay $\tau_{d}$ can significantly alter information transmission by single neurons. It was found previously that delays could cause resonances in the coherence and mutual information rate in networks of neurons that may be exploited by sensory systems [12]. However, the model used was a renewal process that did not display any intrinsic noise shaping. More generally, addition of a delay $\tau_{d}$ has been shown to cause oscillatory dynamics in neuronal networks [20, 21, 41, 74]. Our results are consistent with these as the power spectra and cross-spectra of the average network activity will depend on the delay (i.e. addition of the delay will cause changes in the dynamics). However, these effects are not seen when one considers the mutual information density which normalizes the cross-spectrum by the power spectrum of the response as was recently reported [42]. As such, these oscillations may or may not affect information transmission depending on whether one considers the average network activity or the activity of a single neuron as was previously pointed out [12]. We note that the notion that higher order neurons simply sum the activity of their afferent neurons is at best questionable given recent results. Moreover, experimental recordings can only be currently achieved from a infinitesimal percentage of neurons in the brain. As such, we believe that our results using single neurons are more likely to be directly applicable to experimental data.

Our results have shown that the addition of a delay can significantly alter the effects of coupling on information transmission for renewal but not nonrenewal neurons. Whereas information increased with increasing coupling for no delay for renewal neurons, a non-zero delay could cause the information to now decrease with increasing coupling. Since the majority of synaptic connections in cortex are excitatory and are associated with a non-zero delay due to finite conduction speeds in neural tissue $[4,35]$, our results imply that such coupling would only decrease information transmission by single neurons if they display renewal dynamics. However, our results also imply that such coupling would increase information transmission by single neurons if they display nonrenewal dynamics. Experimental data has shown that many neurons both peripheral and central can display a single negative ISI correlation at lag one and thus nonrenewal dynamics similar to that shown in our nonrenewal model [7, 17, 24, 36, 37, 39, 43, 61]. Moreover, a recent study has shown that temporal anti-correlations appear to improve signal detection in a manner analogous to that seen for a single negative ISI correlation coefficient at lag one [23]. While the consequences of negative ISI correlations on information transmission by single uncoupled neurons is generally well understood $[9,10,17,27,40,44,52,61]$, our results provide a potential explanation for experimental data showing that these ISI correlations are found in more central neurons $[37,39,43]$ that often make and receive thousands of synaptic connections [4, 35]. Our results also complement 
those of [1] who showed that excitatory coupling will also increase information transmission in networks of nonrenewal neurons when considering the average network activity and thus appear to be robust.

Our results using renewal models are similar to those of others $[12,29,51,59,60]$. However, such studies are carried for infinite size networks or using corrections to the thermodynamic limit [51]. Other studies use approximations for small or large delays [59], or very restrictive forms for the feedback input [60], or have been carried out solely in renewal neurons [12]. In contrast, our approach can be carried out for arbitrary values of the networks size or the delay and can be furthermore applied to nonrenewal processes that can display behaviors that are quite different than those displayed by renewal processes [7, 8, 9, 10, 11, 20, 40, 41, 43, 52].

We note that our nonrenewal model displays zero power at zero frequency and has infinite phase coherence $[1,10,40]$. These features are of course not found experimentally. Nevertheless, our nonrenewal model successfully reproduced and explained the mechanism that increases information transmission in neurons: noise shaping [10,40]. This phenomenon was then verified experimentally [8]. Moreover, predictions made previously as to the opposite effect of the bias $\mu$ on the zero frequency power spectrum in renewal and nonrenewal models appear to agree with experimental results [1]. It is thus likely that our predictions would apply to more realistic neuron models that more closely mimic the negative ISI correlations seen experimentally.

We note that many neurons can display weak positive ISI correlations that decay slowly as a function of lag [43]. Such correlations lead to increased variability in the spike train at long timescales and thus worse signal detection [9, 43, 52, 61]. However, as mentioned above, these effects are mostly seen at time scales that are much longer than those observed in animal behavior [55]. We note however that these positive ISI correlations can interact with a single negative ISI correlation at lag one in order to give a time scale at which spike train variability is minimal $[9,43,52,61]$ which corresponds to the behavioral timescale [55].

In conclusion, our results show that delayed feedback and intrinsic ISI correlations can interact in a non-trivial manner to shape information transmission. Future studies of interactions amongst neurons should therefore consider the effects of intrinsic dynamics as these may have important effects. Future studies could extend our results by considering the effects of positive ISI correlations, distributions of delays, or heterogeneous networks.

\section{Acknowledgements}

This research was supported by CONACyT (O. A. A.) and CIHR, CFI, and CRC (M.J.C).

\section{References}

[1] O. Ávila Åkerberg, M.J. Chacron. Noise shaping in neural populations. Phys. Rev. E, 79 (2009), 011904. 
[2] S. Bahar, J.W. Kantelhardt, A. Neiman, H.H.A. Rego, D.F. Russell, L. Wilkens, A. Bunde, and F. Moss. Long range temporal anti-correlations in paddlefish electroreceptors. Europhys. Lett., 56 (2001), 454-460.

[3] A. Borst, F. Theunissen. Information theory and neural coding. Nat. Neurosci. 2 (1999), 947-957.

[4] V. Braitenberg, A. Schüz. Anatomy of the Cortex. Springer, Berlin, 1991.

[5] A. Bulsara, P. Hänggi, F. Marchesoni, F. Moss, M. Shlesinger. Special Issue for Proceedings of The Nato Advanced Research WorkshopStochastic Resonance in Physics and Biology. J. Stat. Phys., 70 (1993), 1-2.

[6] R.S. Cajal. Histologie du système nerveux de l'Homme et des vertébrés. Paris, Maloine, 1909.

[7] M.J. Chacron, A. Longtin, M. St-Hilaire, L. Maler. Suprathreshold stochastic firing dynamics with memory in P-type electroreceptors. Phys. Rev. Lett., 85 (2000), 1576-1579.

[8] M.J. Chacron, L. Maler, J. Bastian. Electroreceptor neuron dynamics shape information transmission. Nat. Neurosci., 8 (2005), 673-678.

[9] M.J. Chacron, A. Longtin, L. Maler. Negative interspike interval correlations increase the neuronal capacity for encoding time-dependent stimuli. J. Neurosci., 21 (2001), 5328-5343.

[10] M.J. Chacron, B. Lindner, A. Longtin. Noise shaping by interval correlations increases information transfer. Phys. Rev. Lett., 92 (2004), 080601.

[11] M.J. Chacron, B. Lindner, A. Longtin. ISI Correlations and Information Transfer. Fluct. Noise Lett., 4 (2004) L195-L205.

[12] M.J. Chacron, A. Longtin, L. Maler. Delayed excitatory and inhibitory feedback shape neural information transmission. Phys. Rev. E, 72 (2005), 051917.

[13] M.J. Chacron, A. Longtin, L. Maler. The effects of spontaneous activity, background noise, and the stimulus ensemble on information transfer in neurons. Network, 14 (2003), 803-824.

[14] M.J. Chacron, B. Doiron, L. Maler, A. Longtin, J. Bastian. Non-classical receptive field mediates switch in a sensory neuron's frequency tuning. Nature, 423 (2003), 77-81.

[15] M.J. Chacron, L. Maler, J. Bastian. Feedback and feedforward control of frequency tuning to naturalistic stimuli. J. Neurosci., 25 (2005), 5521-5532.

[16] M.J. Chacron. Nonlinear information processing in a model sensory system. J. Neurophysiol., 95 (2006), 2933-2946.

[17] M.J. Chacron, B. Lindner, A. Longtin. Threshold fatigue and information transfer. J. Comput. Neurosci., 23 (2007), 301-311. 
[18] M.J. Chacron, J. Bastian. Population coding by electrosensory neurons. J. Neurophysiol., 99 (2008), 1825-1835.

[19] T. Cover, J. Thomas. Elements of Information Theory, Wiley, New-York, 1991.

[20] B. Doiron, M.J. Chacron, L. Maler, A. Longtin, J. Bastian. Inhibitory feedback required for network oscillatory responses to communication but not prey stimuli. Nature, 421 (2003), 539-543.

[21] B. Doiron, B. Lindner, A. Longtin, L. Maler, J. Bastian. Oscillatory activity in electrosensory neurons increases with the spatial correlation of the stochastic input stimulus. Phys. Rev. Lett., 93 (2004), 048101.

[22] L.D. Ellis, R. Krahe, C.M. Bourque, R.J. Dunn, M.J. Chacron. Muscarinic receptors control frequency tuning through the downregulation of an A-type potassium current. J. Neurophysiol., 98 (2007), 1526-1537.

[23] T.A. Engel, B. Helbig, D.F. Russell, L. Schimansky-Geier, A.B. Neiman. Coherent stochastic oscillations enhance signal detection in spiking neurons. Phys. Rev. E, 80 (2009), 021919.

[24] F. Farkhooi, M.F. Strube-Bloss, M.P. Nawrot. Serial correlation in neural spike trains: Experimental evidence, stochastic modeling, and single neuron variability. Phys. Rev. E, 79 (2009), 021905.

[25] L. Gammaitoni, P. Hänggi, P. Jung, F. Marchesoni.Stochastic resonance. Rev. Mod. Phys., 70 (1998), 223-287.

[26] L. Glass, M.C. Mackey. From Clocks to Chaos. Princeton Univ. Press, Princeton, 1988.

[27] J.B.M. Goense, R. Ratnam. Continuous detection of weak sensory signals in afferent spike trains: the role of anti-correlated interspike intervals in detection performance. J. Comp. Physiol. A, 189 (2003), 741-759.

[28] C. Gray, W. Singer. Stimulus-specific neuronal oscillations in orientation columns of cat visual cortex. Proc. Natl Acad. Sci. USA, 86 (1989), 1698-1702.

[29] N.B. Janson, A.G. Balanov, E. Schöll. Delayed Feedback as a Means of Control of NoiseInduced Motion. Phys. Rev. Lett., 93 (2004), 010601.

[30] A. V. Holden. Models of the Stochastic Activity of Neurons. Springer, Berlin, 1976.

[31] H. Hollander. The projection from the visual cortex to the lateral geniculate body (LGB). An experimental study with silver impregnation methods in the cat. Exp. Brain Res., 10 (1990), 219-235.

[32] B. Hutcheon, Y. Yarom, Resonance, oscillation and the intrinsic frequency preferences of neurons. Trends Neurosci., 23 (2000), 216-222. 
[33] E.M. Izhikevich Neural Excitability, Spiking, and Bursting. Int. J. Bif. Chaos, 10 (2000), 1171-1266.

[34] H. Kashiwadani, Y.F. Sasaki, N. Uchida, K. Mori. Synchronized oscillatory discharges of mitral/tufted cells with different molecular receptive ranges in the rabbit olfactory bulb. $\mathrm{J}$. Neurophysiol., 82 (1999), 1786-1792.

[35] Z.F. Kisvárday, K.A. Martin, T.F. Freund, Z. Maglóczky, D. Whitteridge, P. Somogyi. Synaptic targets of HRP-filled layer III pyramidal cells in the cat striate cortex. Exp. Brain. Res., 64 (1986), 541-552.

[36] W.R. Klemm, C.J. Sherry. Entropy as an index of the informational state of neurons. Int. J. Neurosci., 15 (1981), 171-178.

[37] H. Korn, P. Faure. Is there chaos in the brain? I. Concepts of nonlinear dynamics and methods of investigation. C. R. Acad. Sci. III, 324 (2003), 773-793.

[38] R. Krahe, J. Bastian, M.J. Chacron. Temporal processing across multiple topographic maps in the electrosensory system. J. Neurophysiol., 100 (2008), 852-867.

[39] M.A. Lebedev, R.J. Nelson. High-frequency vibratory sensitive neurons in monkey primary somatosensory cortex: entrained and nonentrained responses to vibration during the performance of vibratory-cued hand movements. Exp. Brain Res., 111 (1996), 313-325.

[40] B. Lindner, M.J. Chacron, A. Longtin. Integrate-and-fire neurons with threshold noise: a tractable model of how interspike interval correlations affect neuronal signal transmission. Phys. Rev. E, 72 (2005), 021911.

[41] B. Lindner, B. Doiron, A. Longtin. Theory of oscillatory firing induced by spatially correlated noise and delayed inhibitory feedback. Phys. Rev. E, 72 (2005), 061919.

[42] B. Lindner, D. Gangloff, A. Longtin, J.E. Lewis. Broadband Coding with Dynamic Synapses. J. Neurosci., 29 (2004), 2076-2087.

[43] S.B. Lowen, M.C. Teich. Auditory-nerve action potentials form a nonrenewal point process over short as well as long time scales. J. Accoust. Soc. Am., 92 (1992), 803-806.

[44] N. Lüdtke, M.E. Nelson. Short-term synaptic plasticity can enhance weak signal detectability in nonrenewal spike trains. Neural Comput., 18 (2006), 2879-2916.

[45] K. MacLeod, G. Laurent. Distinct mechanisms for synchronization and temporal patterning of odor-encoding neural assemblies. Science, 274 (1996), 976-979.

[46] Z.F. Mainen, T. J. Sejnowski. Reliability of spike timing in neocortical neurons. Science, 268 (1995), 1503-1506. 
[47] L. Maler, E. Mugnaini. Organization and function of feedback to the electrosensory lateral line lobe of gymnotiform fish, with emphasis on a searchlight mechanism. J. Comp. Physiol. A, 173 (1993), 667-670.

[48] L. Maler, E. Mugnaini. Correlating gamma-aminobutyric circuits and sensory function in the electrosensory lateral line lobe of a gymnotiform fish. J. Comp. Neurol., 345 (1994), 224252.

[49] D.J. Mar, C.C. Chow, W. Gerstner, R.W. Adams, J.J. Collins. Noise shaping in populations of coupled model neurons. Proc. Natl. Acad. Sci., 96 (1999), 10450-10455.

[50] G. Marsat, G.S. Pollack. Effect of the temporal pattern of contralateral inhibition on sound localization cues. J. Neurosci., 25 (2005), 6137-6144.

[51] M. Mattia, P. Del Giudice. Finite-size dynamics of inhibitory and excitatory interacting spiking neurons. Phys. Rev. E, 70 (2004), 052903.

[52] J.W. Middleton, M.J. Chacron, B. Lindner, A. Longtin. Firing statistics of a neuron model driven by long-range correlated noise. Phys. Rev. E, 68 (2003), 021920.

[53] B.A. McGuire, J.P. Hornung, C.D. Gilbert, T.N. Wiesel. Patterns of synaptic input to layer 4 of cat striate cortex. J. Neurosci., 4 (1984), 3021-3033.

[54] F. Moss, L. Ward, W. Sannita. Stochastic resonance and sensory information processing: a tutorial and review of application. Clin. Neurophysiol., 115 (2004), 267-281.

[55] M.E. Nelson, M.A. MacIver. Prey capture in the weakly electric fish Apteronotus albifrons: sensory acquisition strategies and electrosensory consequences. J. Exp. Biol., 202 (1999), 1195-1203.

[56] S.R. Norsworthy, R. Schreier, G. C. Temes. Delta-Sigma Data Converters. IEEE Press, Piscataway, 1997.

[57] E.M. Ostapoff, D.K. Morest, S.J. Potashner. Uptake and retrograde transport of [ $3 H] G A B A$ from the cochlear nucleus to the superior olive in the guinea pig. J. Chem. Neuroanat., 3 (1990), 285-295.

[58] C.L. Passaglia, J.B. Troy. Information transmission rates of cat retinal ganglion cells. J. Neurophysiol., 91 (2004), 1217-1229.

[59] A. Pototsky, N. Janson. Excitable systems with noise and delay, with applications to control: Renewal theory approach. Phys. Rev. E, 77 (2008), 031113.

[60] T. Prager, H.P. Lerch, L. Schimansky-Geier, E. Schöll. Increase of coherence in excitable systems by delayed feedback. J. Phys. A , 40 (2007), 11045-11055. 
[61] R. Ratnam, M.E. Nelson. Nonrenewal statistics of electrosensory afferent spike trains: implications for the detection of weak sensory signals. J. Neurosci., 20 (2000), 6672-6683.

[62] F. Rieke, D. Warland, R.R. de Ruyter van Steveninck, W. Bialek. Spikes: Exploring the Neural Code. MIT press, Cambridge, MA, 1996.

[63] H. Risken. The Fokker-Planck Equation. Springer, Berlin, 1996.

[64] J.C. Roddey, B. Girish, J.P. Miller. Assessing the performance of neural encoding models in the presence of noise. J. Comput. Neurosci., 8 (2000), 95-112.

[65] S. Sadeghi, M.J. Chacron, M.C. Taylor, K.E. Cullen. Neural variability, detection thresholds, and information transmission in the vestibular system. J. Neurosci., 27 (2007), 771-781.

[66] A.M. Sillito, H.E. Jones, G.L. Gerstein, D.C. West. Feature-linked synchronization of thalamic relay cell firing induced by feedback from the visual cortex. Nature, 369 (1994), 479-482.

[67] R. Shannon. The mathematical theory of communication. Bell. Syst. Tech. J., 27 (1948), 379$423,623-656$.

[68] S.M. Sherman. Tonic and burst firing: dual modes of thalamocortical relay. TINS, 24 (2001), $122-126$.

[69] S.M. Sherman, R.W. Guillery. The role of the thalamus in the flow of information to the cortex. Philos. Trans. R. Soc. Lond. B Biol. Sci., 357 (2002),1695-1708.

[70] J. Shin. Adaptation in spiking neurons based on the noise shaping neural coding hypothesis. Neural Networks, 14 (2001), 907-919.

[71] N.G. Stocks. Suprathreshold stochastic resonance in multilevel threshold systems. Phys. Rev. Lett., 84 (2000), 2310-2313.

[72] M. Stopfer, S. Bhagavan, B.H. Smith, G. Laurent. Impaired odour discrimination on desynchronization of odour-encoding neural assemblies . Nature, 390 (1997), 70-74.

[73] A.M. Yacomotti, M.C. Eguia, J. Aliaga, O.E. Martinez, G.B. Mindlin. Interspike Time Distribution in Noise Driven Excitable Systems. Phys. Rev. Lett., 83 (1999), 292-295.

[74] M.K.S. Yeung, S.H. Strogatz. Time Delay in the Kuramoto Model of Coupled Oscillators. Phys. Rev. Lett., 82 (1999), 648-651.

[75] K. Wiesenfeld, I. Satija. Noise tolerance of frequency-locked dynamics. Phys. Rev. B, 36 (1987), 2483-2492. 